\title{
Charlotte Maria
}

Université de Caen-Basse Normandie

\section{Claude Cahun, du rêve à la Résistance}

$D$ our Claude Cahun, la création artistique, sous arts plastiques, théâtre, a toujours été une forme de résistance. Résistance à la douleur, à la folie, à la mort, dans un premier temps, mais aussi résistance aux idées reçues, aux préjugés, à la morale bourgeoise. Devant l'impossibilité de traiter l'ensemble de ces points, le choix s'est porté sur sa dimension politique. Cette résistance de Claude Cahun, I'histoire lui a même attribué un grand " R ». Mais sous ses grands airs, elle fut particulière, fantaisiste, ludique et humaniste.

Pour comprendre comment elle en arriva à ce statut $d^{\prime}$ " " héroïne ", nous nous attacherons principalement à la correspondance de Claude Cahun, en grande partie encore inédite ${ }^{1}$. L'attention se portera dans un premier temps sur son parcours politique. Dans un deuxième temps, elle se concentrera sur la nature des activités politiques de Claude Cahun pendant la Seconde Guerre mondiale. Et enfin, elle ouvrira un questionnement sur le rôle de l'art dans la révolution.

\section{Parcours politique de Claude Cahun}

$S^{\prime}$ il a été tout d'abord question de l'importance du politique dans l'œuvre de Claude Cahun, il faut s'attarder sur le développement de cette prise de conscience. C'est d'abord en tant que juive que Claude Cahun fut confrontée à la réalité politique. Les persécutions dont

\footnotetext{
${ }^{1}$ Une publication est à paraître prochainement.
} 
elle fut l'objet à l'école au moment de la réouverture du procès Dreyfus l'amenèrent à se questionner. Ses premières années furent marquées par une révolte influencée par les sympathies anarchistes de son oncle Marcel Schwob. Mais elle reste alors dans une démarche symboliste qui, par son opposition au naturalisme, apparaît comme uniquement concernée par l'art, et, comme telle, cherche à dégager la littérature de tout intérêt documentaire, de tout rôle social, de toute responsabilité morale.

La révolte de la première jeunesse de Claude Cahun s'accompagne alors d'une sorte de détachement aristocratique. Elle adopte une position extérieure, toute action semblant vaine. Dans son premier livre, Vues et visions, le monde semble lointain, le moi est au-dessus de cette réalité à laquelle il ne semble pas avoir accès : " Je pense au Christ, à son sacrifice inutile, à ses vaines paroles de bonté ${ }^{2}$. L'idée de vanité est en effet présente à plusieurs reprises, exprimant l'idée de l'impossibilité de changer quoi que ce soit :

Vaine offrande. Ce n'est pas d'aujourd'hui, fervent jeune homme, que les dieux sont insensibles. Ce n'est pas d'aujourd'hui et ce n'est pas d'hier.

Ton espérance est vaine, tes prières inutiles, ils ne t'entendent pas ; non sans raison, l'homme impie fait leurs statues et leurs temples de pierre. Les dieux ne s'inquiètent guère des mortels. Pour t'en convaincre, songe seulement aux crimes, aux sacrilèges impunis. ${ }^{3}$

En 1931, elle écrit au comédien Roger Roussot : " Ma destinée est de rester en dehors des entreprises humaines $»^{4}$.

L'engagement politique implique un certain recul par rapport à ses propres démons. Prendre position par rapport au monde, c'est nécessairement sortir de soimême, porter son regard en dehors de ses préoccupations

${ }^{2}$ C. Cahun, Vues et visions, illustrations de Marcel Moore, Paris, Georges Crès et Cie, 1919, p. 71.

3 lbid., p. 79.

${ }^{4}$ Lettre de C. Cahun à Roger Roussot du 19 avril 1931, collection particulière. 
personnelles. Claude Cahun eut longtemps de grandes difficultés à s'abstraire des difficultés liées à sa santé ou aux problèmes matériels :

Depuis notre retour, je ne puis me remettre à rien. Trop de désordre dans la maison dont je ne puis m'abstraire, trop de soucis matériels et trop de sollicitations au-dehors... Au fond, surtout, et comme toujours en moi, le perpétuel "qu'importe ? " Mais qu'importe en effet. Les autres se chargent si bien (parfois, je m'indigne, si mal - mais l'indignation me suffit comme réaction) d'agir à ma place. ${ }^{5}$

Pendant longtemps, il semble que Claude Cahun se sentit submergée par un grand nombre de désagréments qui la maintinrent dans des préoccupations autocentrées. Et pourtant, en 1932, Claude Cahun finit par s'impliquer dans l'Association des Écrivains et Artistes Révolutionnaires (A.E.A.R.) auprès des surréalistes. Elle signa, avec les surréalistes, deux tracts de l'A.E.A.R. : "Protestez " ${ }^{6}$ en mars 1933, qui dénonçait le fascisme en Allemagne et appelait à " un front uni de tous les travailleurs pour venir en aide au prolétariat allemand " et, au mois de mai de la même année, " [c]ontre le fascisme, mais aussi contre l'impérialisme français! ${ }^{7}$, qui dénonçait " l'unité de but de tous les exploiteurs capitalistes, de l'impérialisme français comme du fascisme allemand ${ }^{8}$.

En 1934, solidaire avec les positions d'André Breton, Claude Cahun se fait exclure de l'A.E.A.R. avec les autres membres du Groupe. Elle décide alors de préciser sa position par rapport à l'" Affaire Aragon " et les incompatibilités entre la politique du Parti communiste et le surréalisme, ou du moins sa propre vision de la poésie, en publiant Les paris sont ouverts, plaquette citée par

\footnotetext{
${ }_{5}$ Brouillon d'une lettre C. Cahun à Henri Michaux [1931], collection particulière.

6 "Protestez!", Feuille rouge, n 2, mars 1933, repris [dans :] C. Cahun, Écrits, François Leperlier (éd.), Paris, J.-M. Place, 2002, p. 545-546.

7 " Contre le fascisme mais aussi contre l'impérialisme français ", Feuille rouge, n 4, mai 1933, repris dans C. Cahun, Écrits, op. cit., p. 547.

8 Ibidem.
} 
Breton dans Qu'est-ce que le surréalisme ?9, qui la place au cœur du débat littéraire et politique qui agite l'extrême gauche et le surréalisme.

Mais, jusque là, même si ses positions sont courageuses et à contre-courant de la montée des idées réactionnaires de la France des années trente, l'activité politique de Claude Cahun se limite à la participation à des réunions et à la signature de tracts. Toutefois, tout changera après son installation à Jersey.

\section{L'action « surréaliste-militante »}

Avant que ma plume soit envolée, j'oserai vous parler de notre aventure qui, sur le plan jersiais, ne fut point exempte d'humour noir. Comment vous en exposer la drôlerie ? Il faudrait d'abord que vous sachiez que nous avons eu le privilège de nous livrer pendant quatre ans à une activité surréaliste-militante, du genre de celle que j'imaginais à l'époque de "Contre-Attaque ». Le sacrilège n'y manquait pas, et sous les voûtes de ces déserts de pierre que nous fréquentâmes parfois, non sans bons motifs, la répercussion de nos pas et de nos rires étouffés suffisait à évoquer la présence fraternelle de Benjamin Péret. ${ }^{10}$

Dans cette lettre à Breton, Claude Cahun adopte un ton de légèreté qui est en décalage avec ce qu'elle vient de vivre. La question rhétorique ajoute à cette retenue : les effets sont calculés, toute gravité est laissée de côté, l'aventure est présentée comme un divertissement, presque une bouffonnerie. Pourtant, Claude Cahun paya cher son engagement : elle fut emprisonnée neuf mois dans une prison militaire allemande et condamnée à mort, mais non exécutée.

Dans une autre lettre Claude Cahun raconte la montée de sa révolte et explicite alors les conditions qui l'amènent à l'idée de s'adresser directement aux soldats allemands :

\footnotetext{
${ }^{9}$ A. Breton, Qu'est-ce que le surréalisme? (1934), dans Idem, CEuvres complètes, Paris, Éditions Gallimard, 1992, t. 2, p. 260.

10 Lettre de C. Cahun à André Breton du 18 janvier 1946, Bibliothèque littéraire Jacques-Doucet, [BRT.esup.156].
} 
Je ne sais trop pourquoi je demandai soudain à Suzanne, le lendemain de l'arrivée officielle des "visitors ${ }^{11}$, de me chercher dans nos bibliothèques un numéro du Crapouillot dont j'avais un vague souvenir... Oui. Exact : II y avait quelques documents sur l'Allemagne (pré)hitlérienne. Très peu. Une phrase pourtant... qui m’obséda aussitôt. C'était : "Schrecken ohne Ende oder Ende mit Schrecken! " ohne Ende ohne Ende ohne ${ }^{12}$ se martelait dans ma tête comme ces refrains de roues sur les rails... " Comment dit-on guerre en allemand ? demandai-je à Suzanne. - "Krieg » [...] Suzanne a su parler allemand avant de savoir parler français, mais croyait avoir tout oublié... elle m'assura qu'elle ne savait construire cette simple expression : GUERRE-SANS-FIN...J'envoyai toute grammaire au diable. Je sortis. Je ramassai là-haut, sur la route Orange [sic] des cartons à cigarettes allemands... des verts avec un 5 dessus (devint plus tard un V). J'écrivis au crayon encre (violette, rouge, verte... pour attirer l'attention... sur le revers blanc des cartons que je ramassai l'un après l'autre) :

\section{KRIEG ::: ohne Ende}

... et je posai un à un mes cartons sur petits murs et talus, aussi visibles que je pus. [...] L'idée, la bonne, était là : Leur dire dans leur langage ce que je sentais - qu'il sentait tout comme moi, j'en étais sûre. Mon désespoir était ou serait le leur. Celui d'un des leurs en tous cas. Je le créai à mon image. Dès cette nuit-là je lui donnai un nom : " der Soldat ohne Namen » (...und seine Kamaraden... plus tard...). ${ }^{13}$

Le passage de la théorie à la praxis s'accompagne d'un renoncement à la grammaire qui est abandonnée " au diable ». Par contre, en bonne artiste-propagandiste, le souci de la présentation est extrême : attention portée à la casse des lettres, choix du support, de la couleur de l'encre. Ses messages sont d'abord destinés aux soldats allemands. Notons que cette préoccupation de s'adresser aussi aux Allemands est un fait rare dans l'histoire de la Résistance. Claude Cahun est persuadée qu'elle peut transmettre son désespoir et démoraliser ainsi les soldats

\footnotetext{
${ }^{11}$ Nom ironique donné par les Jersiais aux soldats allemands. Ce mot désigne habituellement les touristes.

12 Citation approximative du proverbe allemand " Lieber ein Ende mit Schrecken als ein Schrecken ohne Ende " (plutôt une fin effroyable qu'un effroi sans fin) utilisé dans le Dix-huit Brumaire de Louis Bonaparte de Karl Marx [1851], à propos de l'arrivée au pouvoir de Napoléon III : "Plutôt une fin effroyable qu'un effroi sans fin!".

13 Lettre de C. Cahun à Charles-Henri Barbier du 21 janvier 1951, collection particulière.
} 
qui occupent Jersey. Pour donner du poids à ses tracts, elle en rédige dans de nombreuses langues, afin de laisser croire qu'une organisation internationale est à l'origine des papiers. Ce sont alors des poèmes et des Bulletins surréalistes qui servent de modèles :

Il est plus singulier que j'aie pu employer d'autres langues inconnues : le russe, le grec, l'espagnol - des bouts de documents, quelques mots extraits de livres classiques, de poèmes devenaient soudain les mots justes du moment. II est moins surprenant que j'aie pu écrire (écrire si l'on peut dire !) un manifeste de défaitisme révolutionnaire à l'intention des Tchèques, ouvriers et soldats, importés ici par les armées allemandes. J'avais pour m'y aider un numéro du Bulletin international du Surréalisme. La traduction me permit de repérer les phrases et de les lier suffisamment. ${ }^{14}$

Sa contre-propagande ne s'adresse pas seulement aux soldats allemands. Beaucoup de tracts sont réalisés pour les ouvriers des Todt et les étrangers de l'armée allemande :

Les bouts de langues " étrangères " s'adressaient aux travailleurs O.T. (pour qui nous avions des textes spéciaux), d'une part, et d'autre part aux étrangers incorporés par la Wehrmacht et plus ou moins repérés par nous à l'aspect. Parmi ces derniers, nous avions repéré des types que nous supposions roumains. Nous les haïssions (mais je n'avais pas de textes en roumain). Suzanne me vengea d'eux - qui ne $m$ 'avaient rien fait que d'afficher leur nazisme plus que les Allemands: ils saluaient à l'hitlérienne - en trouvant le moyen, dans l'autobus, de glisser nos papiers dans la serviette de cuir d'un de ces types. ${ }^{15}$

Pour Claude Cahun, la démarche est aussi artistique que politique. Tous les tracts sont minutieusement réalisés. Les couleurs sont soigneusement choisies. Elle met en pratique son intelligence de l'image, son sens de la mise en scène :

Une page d'illustré allemand s'était trouvée sous mes yeux au cours de nos promenades. Je ramassai. [...] Sur cette page une photographie de régiment en marche. Ça avait l'air plein d'ardeur. Je la tournai en tous sens. Je m'aperçus qu'il suffisait de cacher la moitié de la photo pour changer complètement l'impression qu'elle donnait. Les jambes, les

\footnotetext{
${ }^{14}$ Lettre de C. Cahun à André Breton du 18 janvier 1946, op. cit.

15 Lettre de C. Cahun à Gaston Ferdière de mars 1946, collection particulière.
} 
bottes, (sans les visages) n'avaient rien, rien qui pût sembler exaltant. Elles étaient tachées de boue [...] et, isolées du reste, fatiguées à l'extrême. Je coupai soigneusement la photo. À la peinture rouge je traçai par dessus notre ritournelle et cherchai une présentation esthétique. Je trouvai un joli cadre à la taille voulue. [...] J'ouvris le cadre, substituai la photo de bottes à la photo de 1892 et recollai. ${ }^{16}$

Claude Cahun se livre ici à un exercice de sémiotique de l'image qui lui permet de retourner la propagande nazie. La réalisation du tract suit avec une application professionnelle : découpe "soigneuse ", peinture, cadre. Mais le projet n'en reste pas là. Après avoir mis au point une présentation recherchée, il faut exposer son œuvre, réfléchir à l'endroit le plus propice :

II restait l'anneau pour accrocher au mur. Je savais déjà ce que je voulais en faire. Je savais, depuis le matin, par Edna qu'une maison d'évacué [...] allait être occupée, ne l'était pas encore. Il fallait s'y introduire. Ainsi les soldats trouveraient le " tableau $»{ }^{17}$

Le collage, peint et encadré, acquiert désormais le titre de " tableau ". Les guillemets suggèrent certes une certaine distance, mais l'utilisation de ce mot souligne l'implication esthétique. Mais Claude Cahun va encore plus loin et réfléchit à l'interaction avec son " public »:

Mieux valait qu'ils ne le trouvent pas dès l'arrivée ; ainsi chacun pourrait croire qu'il était l'œuvre d'un des autres. [...] Je décidai Suzanne. Ce fut elle qui trouva à l'entrée, une sorte de cave à soupirail, vide et propre. Un clou bien placé. L'éclairage du soupirail tombait en plein sur l'image. Tout se passa sans incident et nous avons pu apercevoir - de loin - les occupants de cette maison quelques jours plus tard. ${ }^{18}$

On remarque l'attention particulière à la lumière et le soin avec lequel elle organise la meilleure scénographie possible pour obtenir l'effet recherché sur le "spectateur».

Parmi les productions les plus sophistiquées figure la réalisation de "vrais-faux " magazines allemands. À partir des revues allemandes en vente dans les librairies,

\footnotetext{
16 Ibidem.

17 Ibidem.

18 Ibidem.
} 
elle réalise des détournements par le biais de subtils photomontages dont elle raconte en détail la réalisation :

J'achetai des illustrés en ville - les cachant, pour ne pas donner le mauvais exemple aux civils. [...] À la maison je coupais et collais, cherchant des photomontages significatifs (à mes yeux ?) et aussi à apprendre un peu d'allemand. J'avais grand mal à feuilleter tout ça c'est même impossible à exprimer à quel point - jusqu'au moment où quelque chose à en faire me sautait aux yeux. Alors, m'en servir devenait une obsession et en somme une sorte de plaisir technique. ${ }^{19}$

Claude Cahun apporte ici une description intéressante du processus de création vu comme le caractère saillant, dans une masse informe, d'un élément manifeste qui devient un motif entêtant dont on ne peut plus se défaire.

Une fois le travail technique achevé, il faut trouver un moyen de distribution. Claude Cahun décide alors de remettre les revues en circulation dans les magasins :

Les collages prêts, nous retournions en ville. En achetant de nouveaux illustrés, je feuilletais et introduisais les feuillets de ma propagande. Ce n'était pas facile sous les yeux des clients et commerçants. Le meilleur moyen était d'acheter un illustré dans une boutique, d'aller à la pâtisserie (aux w.c.), d'arranger mes feuillets à loisir dans ce journal. Puis d'aller dans une autre librairie, sous prétexte d'acheter un illustré et d'y déposer le mien - la couverture pareille aux autres. ${ }^{20}$

Les supports de sa propagande sont extrêmement variés. Elle réalise même, avec l'aide de Suzanne, un Lied aux paroles détournées ainsi que d'autres objets artistiques de propagande. On retrouve notamment un calendrier "divinatoire " qui donne à son goût pour l'occultisme une finalité surprenante :

Une sorte de calendrier. On tirait une bande dissimulée entre deux cartons, et par une "fenêtre " on lisait la " bonne " (!) aventure du soldat envoyé en Russie. Sur la face externe du carton un dessin caricatural en couleur. C'était plus difficile à placer - mais quelques occasions se présentèrent. ${ }^{21}$

\footnotetext{
19 Ibidem.

${ }^{20}$ Ibidem.

21 Ibidem.
} 
Claude Cahun donne libre cours à son imagination, et alors qu'elle ne publiait plus depuis des années, qu'elle semblait limitée dans ses travaux d'écriture par le poids des traditions familiales, le "plaisir technique " de la réalisation et le contexte de guerre impliquant un caractère à la fois ludique par la nécessité d'être caché et sérieux par la dimension politique du message lui ouvre de nouveaux horizons et débride son sens créatif :

Il y eut la période des sous peints. Je les peignais minutieusement avec des vernis à ongles - il me restait des fonds de flacons fort variés - et parvenais à inscrire très clairement dessus NIEDER MIT KRIEG ${ }^{22}$. Nous en disposâmes aisément. Il y avait en ville un " amusement park "23, fréquenté presque exclusivement par des soldats et marins allemands. Nous y entrâmes à une heure où il n'y avait que les tenanciers. Puis blaguant entre nous en anglais, nous fîmes marcher un ou deux appareils y laissant nos sous à slogan. Ce fut même avantageux la première fois ! Je vernis notre gain et l'y ajoutais. ${ }^{24}$

\section{Art et révolution}

La dimension politique semble avoir décuplé son inspiration. Les contraintes liées aux conditions matérielles nourrissent l'imagination et donnent lieu à de nombreuses innovations esthétiques au-delà de la simple agit-prop. D'ailleurs Claude Cahun a-t-elle eu accès aux créations qui se font en URSS dès les années vingt ? Sa participation à I'AEAR, sa proximité avec Breton et des membres de l'opposition de gauche dénotent un intérêt pour ce qu'il pouvait se passer en Union soviétique. Et en effet, l'œuvre de propagande d'Alexandre Rodchenko pour la photographie ou Dziga Vertov pour le cinéma donne corps à un projet artistique qui est à la fois un message politique et une exploration esthétique de leur médium :

Dziga Vertov voulait capturer la trace de la vie, afin de la transcender à l'aide du film : de la matière à la vision. [...] L'extension apparemment

\footnotetext{
${ }^{22}$ En allemand dans le texte : " $\mathrm{A}$ bas la guerre ».

${ }^{23}$ En anglais dans le texte : "Parc d'attractions ».

24 Lettre de C. Cahun à Gaston Ferdière de mars 1946, op. cit.
} 
infinie dans le cinéma et la fragmentation s'oppose à la possibilité de synthèse, de rythmicité par le montage. C'est ce " je vois " sous forme d'extrait, l'expérience de la vision, qui caractérise l'équilibre explosif de la vision et de la pensée (le Ciné-CEil) dans la modernité. ${ }^{25}$

Si l'on connaît les limites que la politique stalinienne imposa à l'art, on peut cependant constater l'élan créatif qui s'empara de toute une génération après la révolution de 1917. Les possibilités contenues en germe dans cette transformation radicale de la société semblent avoir donné aux artistes, dans un premier temps, un regard nouveau sur l'art.

Comme art politique, la question de la destination des $œ u v r e s$ est primordiale. Ainsi Claude Cahun est très préoccupée de la "réception " de ses objets : "Lors du procès, le Conseil de Guerre en avait en mains quelquesuns [des sous peints] - un faible pourcentage. Le reste fut, je l'espère, gardé par les marins allemands $\$^{26}$. Elle s'inquiète de leur destination, de leur succès. La démarche est artistique, mais l'objectif reste politique. Refusant I'inaction, il s'agit de dénoncer la guerre, de toucher tous ceux qui sont démoralisés, y compris les soldats allemands. La méthode choisie, pour le moins originale, est justifiée dans une lettre à André Breton :

Contre un monstre à plusieurs faces, seules les armes à plusieurs tranchants peuvent être efficaces, et si l'analogie ambitieuse des cristaux ne venait au secours de qui les emploie, l'estime de soi-même risquerait de sombrer. ${ }^{27}$

La pratique militante de Claude Cahun à Jersey rejoint les conceptions exposées dans sa brochure Les paris sont ouverts. Elle en résume l'objet dans une lettre à sa nièce :

Ce pamphlet était une défense de la poésie libre (celle des surréalistes) que j'opposais à la poésie de propagande - ou comme on dit aujourd'hui " engagée » (celle d’Aragon reniant ses antécédents surréalistes). En

25 O. Fahle, "L'œil pointé sur la modernité », [dans :] Esquenazi J.-P. (dir.), VERTOV - L'invention du réel !, Paris, Éditions L'Harmattan, 1998, p. 146.

${ }^{26}$ Lettre de C. Cahun à Gaston Ferdière de mars 1946, op. cit.

27 Lettre de C. Cahun à André Breton du 18 janvier 1946, op. cit. 
rédigeant cette brochure, je fus amenée à étudier, par-deçà et par-delà la valeur poétique des textes que je citais, leur valeur de propagande. ${ }^{28}$

En effet, dans Les paris sont ouverts, Claude Cahun prônait " l'action politique indirecte ". Pour elle, l'efficacité de la propagande tient dans cette distance instaurée par la démarche artistique. Claude Cahun revendiqua par ailleurs la dimension esthétique de sa propagande qu'elle-même considérait comme faisant partie de son travail d'écrivain :

Mon analyse des méthodes plus ou moins efficaces de propagande m'a beaucoup servi en 1940 à 1944 . Tout m'a prouvé que la propagande antinazie défaitiste - mon travail « littéraire » de ces années-là - avait été efficace. ${ }^{29}$

On voit ici se confondre la démarche plastique, littéraire et militante dans un seul et même mouvement qui supprime les frontières entre l'art, l'histoire et la vie. Pour Claude Cahun, le besoin de défendre ses idées par tous les moyens apparaît tout au long de ses lettres qui visent autant à dénoncer qu'à montrer des moyens de lutte.

\section{Conclusion}

Claude Cahun, comète du mouvement surréaliste, s'est toujours tenue dans les marges, même si elle s'est tenue quelque temps très proche de la figure solaire de Breton. Fidèle à son excentricité, elle a mené un parcours tout à fait original, du retrait aristocratique à l'engagement politique corps et biens :

Si jamais un lecteur me tend la main, ce ne saurait être qu'une main fière. Il me sait révoltée, il me sait asociale - une rêveuse évolutionnaire $[\mathrm{sic}] \ldots{ }^{30}$

28 Lettre de C. Cahun à Marianne Schwob du 18 août 1948, Bibliothèque municipale de Nantes, [Ms 3412/3].

${ }^{29}$ Ibidem.

30 Brouillon incomplet d'une lettre de C. Cahun à Jean Legrand [fin 1945], Jersey Heritage Trust, [JHT/1995/00045/1]. 
Durant toute la période de la Seconde Guerre mondiale et de son activité surréaliste-militante, pour Claude Cahun créer ce fut littéralement Résister. Résister à l'inaction, à l'indifférence, à l'injustice, à la guerre. Si la révolte fut un réel moteur pour la création, son art fut pris très au sérieux par l'armée allemande. Elle fut arrêtée, le 25 juillet 1944, avec sa compagne Suzanne Malherbe, et jugée par un tribunal militaire qui les condamna à mort, sans les exécuter, mais les maintint en prison jusqu'à la Libération de l'île le 9 mai 1945.

Tout en restant unique, Claude Cahun prit part aux événements mondiaux avec imagination et bravoure et montra tout ce que l'art peut faire pour la Révolution. 


\section{bibliographie}

Breton, A., Qu'est-ce que le surréalisme ?, [dans :] Idem, CEuvres complètes, II, éd. Marguerite Bonnet, Paris, Éditions Gallimard, 1992.

Cahun, C., Vues et visions, illustrations de Marcel Moore, Paris, Georges Crès et Cie, 1919.

Esquenazi J.-P. (dir.), VERTOV - L'invention du réel !, Paris, Éditions L'Harmattan, 1998.

[Inédits :]

Lettre à Roger Roussot du 19 avril 1931.

Brouillon d'une lettre à Henri Michaux [1931].

Brouillon incomplet d'une lettre Jean Legrand [fin 1945].

Lettre à André Breton du 18 janvier 1946.

Lettre à André Breton du 18 janvier 1946.

Lettre à Gaston Ferdière de mars 1946.

Lettre à André Breton du 18 janvier 1946.

Lettre à Marianne Schwob du 18 août 1948

Lettre à Charles-Henri Barbier du 21 janvier 1951.

\section{abstract}

Claude Cahun was a writer, photographer, artist, journalist, actress, who was connected with the surrealist group in the interwar period. Living in Jersey with her girlfriend during World War II, she took part in special resistance activities. Isolated from any political group, she had decided to react to the occupation of the island by Nazi Germany with her own artistic propaganda.

\section{keywords}

surrealism, revolution, résistance, World War II

\section{charlotte maria}

Charlotte Maria a soutenu en novembre 2013 une thèse de doctorat en langues et littératures françaises à l'Université de Caen intitulée : "Correspondances de Claude Cahun, la lettre et l'œuvre ». En plus du volume de lettres (en grande partie inédites, en voie de publication), présentées et annotées, la thèse comporte un second volume contenant une étude des lettres et de l'œuvre de Claude Cahun. Ce travail se focalise sur plusieurs axes parmi lesquels se trouvent le positionnement littéraire, la question politique et l'écriture de soi. Elle a également publié une dizaine d'articles sur Claude Cahun dont le dernier est paru au printemps dernier dans la revue en ligne Nouvelle Fribourg sous le titre: "Défiguration et hybridation, l'identité au corps à corps ". 
